\title{
Indium-Catalyzed Direct Conversion of Lactones into Thiolactones Using a Disilathiane as a Sulfur Source
}

\author{
Yohei Ogiwara ${ }^{(D)}$, Ken Takano, Shuhei Horikawa and Norio Sakai* \\ Department of Pure and Applied Chemistry, Faculty of Science and Technology, Tokyo University of Science, \\ 2641 Yamazaki, Noda, Chiba 278-8510, Japan; yoheiogiwara@rs.tus.ac.jp (Y.O.); 7218534@ed.tus.ac.jp (K.T.); \\ 7216665@alumni.tus.ac.jp (S.H.) \\ * Correspondence: sakachem@rs.noda.tus.ac.jp; Tel.: +81-4-7123-9890
}

Received: 16 May 2018; Accepted: 1 June 2018; Published: 2 June 2018

check for updates

\begin{abstract}
An indium-catalyzed reaction of lactones and a disilathiane leading to thiolactones is described. The direct synthesis of thiolactones from lactones with an appropriate sulfur source is one of the most attractive approaches in organic and pharmaceutical chemistry. In this context, we found an indium-catalyzed direct conversion of lactones into thiolactones in the presence of elemental sulfur and a hydrosilane via formation of the disilathiane in situ. On the basis of the previous reaction, the application utilizing the disilathiane as a sulfur source was performed herein for the efficient synthesis of a variety of thiolactone derivatives from lactones by an indium catalyst.
\end{abstract}

Keywords: indium catalyst; disilathiane; lactones; thiolactones

\section{Introduction}

The introduction of a sulfur atom to organic molecules is a significant topic in synthetic chemistry because it potentially provides complex and important sulfur-containing compounds directly. Therefore, a search for an undiscovered sulfur source that could be applicable to organic sulfur chemistry is imperative, and extensive efforts have been devoted to the development of molecular transformations utilizing a novel sulfur source by many research groups thus far [1-6]. In this context, our group has demonstrated that the copper-catalyzed construction of diaryl sulfides from aryl iodides and hexamethyldisilathiane, $\left(\mathrm{Me}_{3} \mathrm{Si}\right)_{2} \mathrm{~S}$ [7]. In the reaction, the disilathiane functioned as an effective S1 source of sulfides, and the results suggested to us that the strategy employing the disilathiane could be acceptable for any other sulfur-introduction reactions [8-12].

Recently, we also reported the indium-catalyzed reductive conversion of lactones 1 into thiolactones 2 using a combination of elemental sulfur $\left(\mathrm{S}_{8}\right)$ and a hydrosilane, wherein the generation of a disilathiane $\left([\mathrm{Si}]_{2} \mathrm{~S}\right)$ from $\mathrm{S}_{8}$ and a hydrosilane is a key process for the formation of thiolactones 2 (Scheme 1a) [13]. Although the in situ formation strategy of the disilathiane is a useful and an easily handled procedure, the yields of thiolactones 2 obtained by the method remained at low to moderate levels. We envisioned that the problem could be overcome by utilizing the activated disilathiane, which can be easily prepared from $\mathrm{S}_{8}$ and a hydrosilane in advance. Herein, we describe the indium-catalyzed direct formation of thiolactones $\mathbf{2}$ from lactones $\mathbf{1}$ using hexamethyldisilathiane as an effective S1 source (Scheme 1b). 
a) previous work

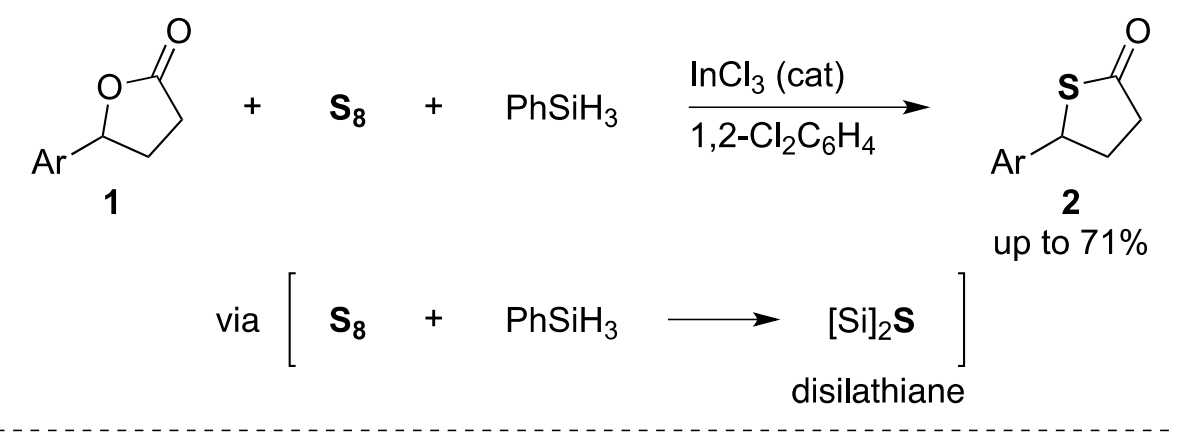

b) this work

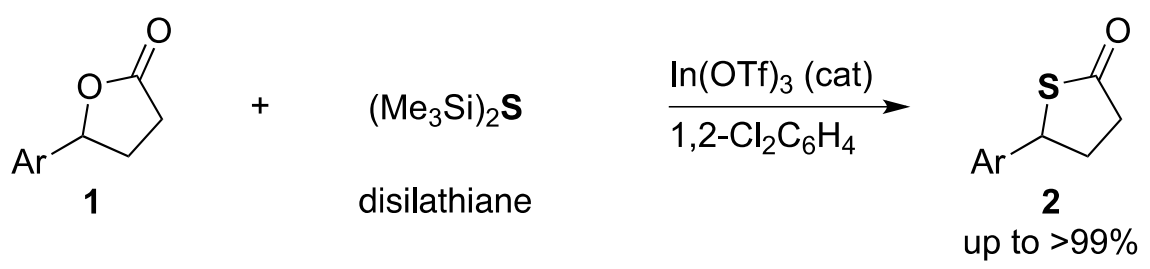

Scheme 1. Indium-catalyzed conversion of lactones 1 to thiolactones 2 using (a) $S_{8}$ and a hydrosilane, and (b) a disilathiane.

\section{Results and Discussion}

On the bases of our previous study on the $\mathrm{InCl}_{3}$-catalyzed transformation of lactones $\mathbf{1}$ to thiolactones 2 employing elemental sulfur $\left(S_{8}\right)$ and a hydrosilane, optimization studies utilizing a disilathiane as a sulfur source were initially conducted (Table 1 ). When $\gamma$-phenyl- $\gamma$-butyrolactone (1a) was treated with 1.1 equiv of hexamethyldisilathiane, $\left(\mathrm{Me}_{3} \mathrm{Si}\right)_{2} \mathrm{~S}$, in the presence of $5 \mathrm{~mol} \%$ of $\mathrm{InCl}_{3}$ in 1,2-dichlorobenzene at $80^{\circ} \mathrm{C}$ for $24 \mathrm{~h}$, the corresponding $\gamma$-butyrothiolactone $2 \mathrm{a}$ was obtained in a 77\% GC yield (entry 1). The formation of $2 \mathbf{a}$ was also observed in cases with other catalysts, such as $\mathrm{InBr}_{3}, \mathrm{InI}_{3}, \mathrm{In}(\mathrm{OAc})_{3}, \mathrm{In}(\mathrm{OTf})_{3}$, and $\mathrm{Cu}(\mathrm{OTf})_{2}$ in good yields (entries 2-6). Especially, $\mathrm{In}(\mathrm{OTf})_{3}$ proved to be the most effective catalyst for the reaction, shown in entry 5, which provided $2 \mathrm{a}$ in a $99 \%$ GC yield with a $94 \%$ isolated yield. In contrast, in the absence of the catalyst, thiolactone was not generated (entry 7). We next chose chlorobenzene, 1,2-dichloroethane, and toluene as potential solvents for the transformation based on our previous results [13]. Although these solvents were also acceptable to the reaction, these yields were not higher than those using 1,2- $\mathrm{Cl}_{2} \mathrm{C}_{6} \mathrm{H}_{4}$ as a solvent (entries 8-10 vs. entry 5). The reaction with a lower catalyst loading (1 mol \% of $\left.\operatorname{In}(\mathrm{OTf})_{3}\right)$ made it possible to form $2 \mathrm{a}$ in a $97 \%$ GC yield with an $83 \%$ isolated yield (entry 11), and then a gram-scale application using 1 a $(5 \mathrm{mmol})$ and $\left(\mathrm{Me}_{3} \mathrm{Si}\right)_{2} \mathrm{~S}(5.5 \mathrm{mmol})$ provided $0.70 \mathrm{~g}(3.9 \mathrm{mmol})$ of thiolactone $2 \mathrm{a}$ (entry 12). Employing a stoichiometric amount of $\mathrm{TfOH}$ instead of $\mathrm{In}(\mathrm{OTf})_{3}$ catalyst provided thiolactone quantitatively (entry 13), whereas its application to the $\mathrm{TfOH}$-catalyzed reaction did not proceed well (entry 14).

Conversion of lactone $\mathbf{1 a}$ into thiolactone $\mathbf{2} \mathbf{a}$, and not into the other possible sulfur-containing compounds, such as the thionolactone $\mathbf{3 a}$ and the dithiolactone $4 \mathbf{a}$, can be easily discriminated by ${ }^{1} \mathrm{H}$ and ${ }^{13} \mathrm{C}-\mathrm{NMR}$ spectroscopy (Table 2 and Figure 1 ). In the ${ }^{1} \mathrm{H}-\mathrm{NMR}$ spectrum of the thiolactone 2a, a signal for the methine proton $(\mathrm{CH})$ next to the oxygen appeared at $5.01 \mathrm{ppm}$ in $\mathrm{CDCl}_{3}$, which is clearly different to those of the corresponding protons of $\mathbf{1 a}(5.52 \mathrm{ppm}), \mathbf{3 a}(5.86 \mathrm{ppm})$, and $4 \mathbf{a}$ (5.29 ppm). The chemical shifts of the carbonyl $(C=\mathrm{O})$ or the thiocarbonyl $(C=S)$ in the ${ }^{13} C-N M R$ spectrum can also identify the difference between 1a (176.9 ppm in $\left.\mathrm{CDCl}_{3}\right), \mathbf{2 a}(207.9 \mathrm{ppm})$, 3a (221.9 ppm), and $4 \mathrm{a}(245.3 \mathrm{ppm})$. For most of the isolated products by this procedure shown below (e.g., Table 3), therefore, these structures were assigned as thiolactone 2 forms by ${ }^{1} \mathrm{H}$ and ${ }^{13} \mathrm{C}-\mathrm{NMR}$ analyses (The detailed spectral data are summarized in Section 3.4. Product Characterization and the Supplementary Materials). 
Table 1. Screening of the reaction conditions for the catalytic conversion of 1 a to $2 a^{a}$.

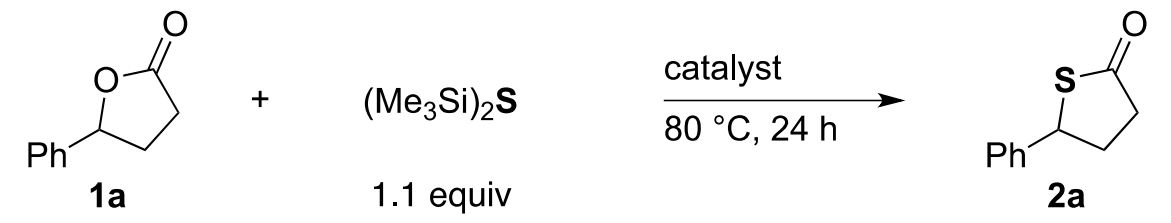

\begin{tabular}{cccc}
\hline Entry & Catalyst & Solvent & GC Yield of 2a \\
\hline 1 & $\mathrm{InCl}_{3}(5 \mathrm{~mol} \%)$ & $1,2-\mathrm{Cl}_{2} \mathrm{C}_{6} \mathrm{H}_{4}$ & 77 \\
2 & $\mathrm{InBr}_{3}(5 \mathrm{~mol} \%)$ & $1,2-\mathrm{Cl}_{2} \mathrm{C}_{6} \mathrm{H}_{4}$ & 62 \\
3 & $\mathrm{InI}_{3}(5 \mathrm{~mol} \%)$ & $1,2-\mathrm{Cl}_{2} \mathrm{C}_{6} \mathrm{H}_{4}$ & 69 \\
4 & $\mathrm{In}(\mathrm{OAc})_{3}(5 \mathrm{~mol} \%)$ & $1,2-\mathrm{Cl}_{2} \mathrm{C}_{6} \mathrm{H}_{4}$ & 78 \\
5 & $\mathrm{In}(\mathrm{OTf})_{3}(5 \mathrm{~mol} \%)$ & $1,2-\mathrm{Cl}_{2} \mathrm{C}_{6} \mathrm{H}_{4}$ & $99(94)^{b}$ \\
6 & $\mathrm{Cu}(\mathrm{OTf})_{2}(5 \mathrm{~mol} \%)$ & $1,2-\mathrm{Cl}_{2} \mathrm{C}_{6} \mathrm{H}_{4}$ & 94 \\
7 & none & $1,2-\mathrm{Cl}_{2} \mathrm{C}_{6} \mathrm{H}_{4}$ & 0 \\
8 & $\mathrm{In}(\mathrm{OTf})_{3}(5 \mathrm{~mol} \%)$ & $\mathrm{ClC}_{6} \mathrm{H}_{5}$ & 76 \\
9 & $\mathrm{In}(\mathrm{OTf})_{3}(5 \mathrm{~mol} \%)$ & $\mathrm{ClCH}_{2} \mathrm{CH}_{2} \mathrm{Cl}$ & 95 \\
10 & $\mathrm{In}(\mathrm{OTf})_{3}(5 \mathrm{~mol} \%)$ & $\mathrm{CH}_{3} \mathrm{C}_{6} \mathrm{H}_{5}$ & 59 \\
11 & $\mathrm{In}(\mathrm{OTf})_{3}(1 \mathrm{~mol} \%)$ & $1,2-\mathrm{Cl}_{2} \mathrm{C}_{6} \mathrm{H}_{4}$ & $97(83)^{b}$ \\
12 & $\mathrm{In}(\mathrm{OTf})_{3}(1 \mathrm{~mol} \%)$ & $1,2-\mathrm{Cl}_{2} \mathrm{C}_{6} \mathrm{H}_{4}$ & $78{ }^{b, c}$ \\
13 & $\mathrm{TfOH}(100 \mathrm{~mol} \%)$ & $1,2-\mathrm{Cl}_{2} \mathrm{C}_{6} \mathrm{H}_{4}$ & 99 \\
14 & $\mathrm{TfOH}(15 \mathrm{~mol} \%)$ & $1,2-\mathrm{Cl}_{2} \mathrm{C}_{6} \mathrm{H}_{4}$ & 23 \\
\hline
\end{tabular}

${ }^{a}$ Reaction conditions: $1 \mathrm{a}(0.5 \mathrm{mmol}),\left(\mathrm{Me}_{3} \mathrm{Si}\right)_{2} \mathrm{~S}(0.55 \mathrm{mmol})$, catalyst $(0.005-0.025 \mathrm{mmol})$, solvent $(0.5 \mathrm{~mL})$ at $80^{\circ} \mathrm{C}$ for $24 \mathrm{~h} .{ }^{b}$ Isolated yield. ${ }^{c} 5$ mmol scale.

Table 2. Selected ${ }^{1} \mathrm{H}$ and ${ }^{13} \mathrm{C}-\mathrm{NMR}$ spectral data $\left(\mathrm{CDCl}_{3}, \mathrm{rt}\right)$ for $\mathbf{1 a}, \mathbf{2 a}, \mathbf{3 a}$, and $\mathbf{4 a}$.

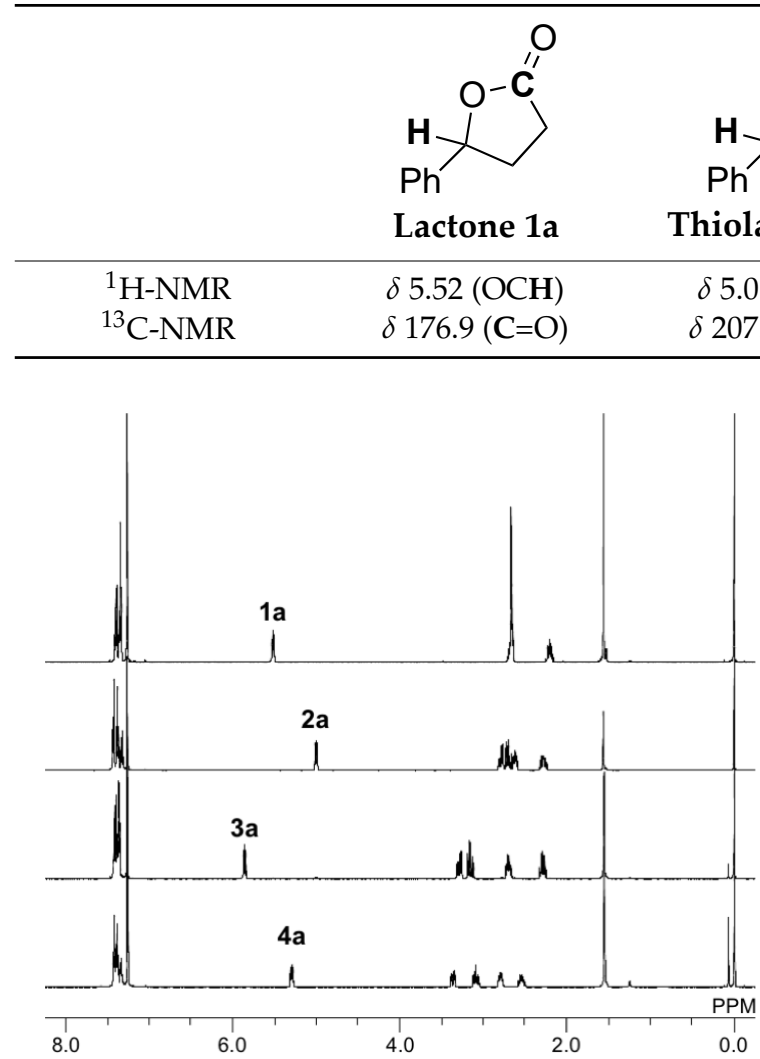

(a)

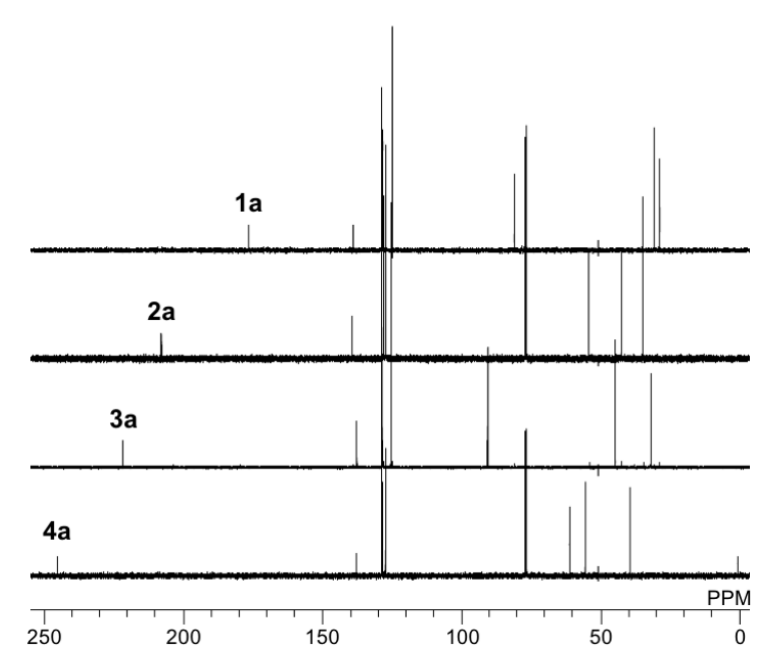

(b)

Figure 1. NMR spectra of 1a-4a: (a) ${ }^{1} \mathrm{H}-\mathrm{NMR}\left(500 \mathrm{MHz}, \mathrm{CDCl}_{3}, \mathrm{rt}\right),(\mathbf{b}){ }^{13} \mathrm{C}-\mathrm{NMR}\left(126 \mathrm{MHz}, \mathrm{CDCl}_{3}, \mathrm{rt}\right)$. 
Molecules 2018, 23, 1339

4 of 11

Table 3. Indium-catalyzed conversion of lactones $\mathbf{1}$ to the thiolactones $\mathbf{2}$.

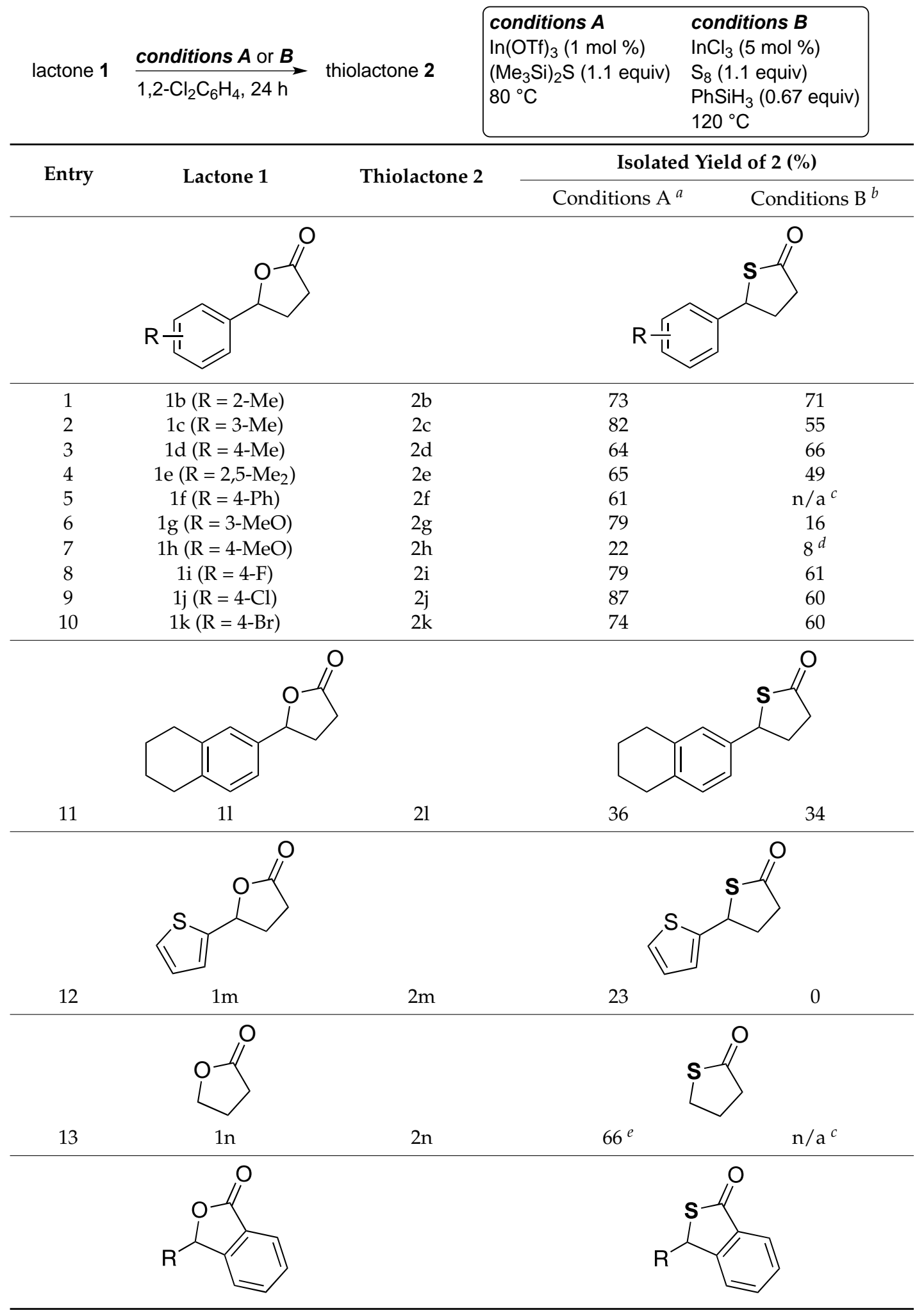


Table 3. Cont.

\begin{tabular}{|c|c|c|c|c|}
\hline lactone 1 & $\underset{1,2-\mathrm{Cl}_{2} \mathrm{C}_{6} \mathrm{H}_{4}, 24 \mathrm{~h}}{\text { conditions }}$ & thiolactone 2 & $\begin{array}{l}\text { conditions } A \\
\ln (\mathrm{OTf})_{3}(1 \mathrm{~mol} \%) \\
\left(\mathrm{Me} \mathrm{S}_{3} \mathrm{Si}\right)_{2} \mathrm{~S}(1.1 \text { equiv }) \\
80^{\circ} \mathrm{C}\end{array}$ & $\begin{array}{l}\text { conditions B } \\
\mathrm{InCl}_{3}(5 \mathrm{~mol} \%) \\
\mathrm{S}_{8}(1.1 \text { equiv) } \\
\mathrm{PhSiH}_{3}(0.67 \text { equiv) } \\
120^{\circ} \mathrm{C}\end{array}$ \\
\hline \multirow{2}{*}{ Entry } & \multirow{2}{*}{ Lactone 1} & \multirow{2}{*}{ Thiolactone 2} & \multicolumn{2}{|c|}{ Isolated Yield of $2(\%)$} \\
\hline & & & Conditions $\mathrm{A}^{a}$ & Conditions $\mathrm{B}^{b}$ \\
\hline 14 & $10(\mathrm{R}=\mathrm{H})$ & 20 & $18^{f}$ & 0 \\
\hline 15 & $1 \mathrm{p}(\mathrm{R}=\mathrm{Ph})$ & $2 p$ & 85 & 10 \\
\hline 16 & $1 \mathrm{q}(\mathrm{Ar}=\mathrm{Ph})$ & $2 q$ & $10 \mathrm{~g}$ & 14 \\
\hline 17 & & $2 \mathrm{r}$ & 9 & $\mathrm{n} / \mathrm{a}^{c}$ \\
\hline 18 & $1 \mathrm{~s}\left(\mathrm{Ar}=4-\mathrm{ClC}_{6} \mathrm{H}_{4}\right)$ & $2 \mathrm{~s}$ & 4 & $\mathrm{n} / \mathrm{a}^{c}$ \\
\hline 19 & $1 \mathrm{t}$ & $2 t$ & $88^{h}$ & 23 \\
\hline
\end{tabular}

${ }^{a}$ This work: $1(0.5 \mathrm{mmol}),\left(\mathrm{Me}_{3} \mathrm{Si}\right)_{2} \mathrm{~S}(0.55 \mathrm{mmol}), \mathrm{In}(\mathrm{OTf})_{3}(0.005 \mathrm{mmol}), 1,2-\mathrm{Cl}_{2} \mathrm{C}_{6} \mathrm{H}_{4}(0.5 \mathrm{~mL})$ at $80{ }^{\circ} \mathrm{C}$ for $24 \mathrm{~h}$.

${ }^{b}$ Previous work: $1(0.5 \mathrm{mmol}), \mathrm{S}_{8}(0.55 \mathrm{mmol}$ of $\mathrm{S}$ atom $), \mathrm{PhSiH}_{3}(0.33 \mathrm{mmol}), \mathrm{InCl}_{3}(0.025 \mathrm{mmol}), 1,2-\mathrm{Cl}_{2} \mathrm{C}_{6} \mathrm{H}_{4}$

$(0.5 \mathrm{~mL})$ at $120^{\circ} \mathrm{C}$ for $24 \mathrm{~h} .{ }^{c}$ Not applicable. ${ }^{d} 4$-(4-Methoxyphenyl)butanoic acid (30\%) was formed. ${ }^{e}$ NMR yield.

$f 7$ d. ${ }^{g} 120{ }^{\circ} \mathrm{C}, 3$ days. ${ }^{h} \mathrm{InCl}_{3}(0.025 \mathrm{mmol}, 5 \mathrm{~mol} \%), 120^{\circ} \mathrm{C}, 20 \mathrm{~h}$.

Examination of the $\operatorname{In}(\mathrm{OTf})_{3}$-catalyzed direct transformation of several lactones 1 was then conducted under the conditions described for entry 10 in Table 1 . The results of the present reaction utilizing an $\mathrm{In}(\mathrm{OTf})_{3} /\left(\mathrm{Me}_{3} \mathrm{Si}\right)_{2} \mathrm{~S}$ system (conditions $\mathrm{A}$ ) and our previous yields of thiolactones 2 by an $\mathrm{InCl}_{3}$-catalyzed reaction using $\mathrm{S}_{8} / \mathrm{PhSiH}_{3}$ (conditions B) [13] are summarized in Table 3 . In most of the substrates, the conditions A showed a better reactivity for the conversion of $\mathbf{1}$ into $\mathbf{2}$ than that of conditions B. Reactions of $\gamma$-aryl- $\gamma$-butyrolactons $\mathbf{1} \mathbf{b}-\mathbf{1 k}$ bearing various functional groups at the aryl ring, such as methyl, phenyl, methoxy, and halogen, afforded the corresponding $\gamma$-aryl- $\gamma$-butyrothiolactones $\mathbf{2 b}-\mathbf{2 k}$ (entries 1-10). Among these entries, the yield of a 4-methoxy-substituted one $\mathbf{2 h}$ was not sufficient (entry 7), probably because of benzylic C-O bond cleavage prior to the reaction with disilathiane due to the strong electron donation by the 4-methoxy substituent. Similar results were also obtained in the case of our previous investigation employing an $\mathrm{S}_{8} / \mathrm{PhSiH}_{3}$ system prominently, which provided 4-(4-methoxyphenyl)butanoic acid as a side product in a $30 \%$ yield (entry 7 , conditions B). A tetralin and a thiophene ring were also acceptable to $\gamma$-aryl substituents of the butyrolactons $\mathbf{1 1}$ and $\mathbf{1 m}$, forming $\mathbf{2 1}$ and $\mathbf{2 m}$ (entries 11 and 12). The simple unsubstituted $\gamma$-butyrolactons $\mathbf{1 n}$, phthalide (10), and its derivative $\mathbf{1 p}$ were converted into the products $\mathbf{2 n - 2} \mathbf{p}$, respectively (entries $13-15)$. When $\delta$-lactones $\mathbf{1} \mathbf{q}-\mathbf{1 t}$ were used as starting substrates, the formation of the expected six-membered thiolactones $\mathbf{2 q - 2 t}$ were observed (entries 16-19).

Finally, the present procedure was evaluated using substrates involving an ester moiety, but not lactones. When phthalic anhydride $(\mathbf{1} \mathbf{u})$ was treated with a disilathiane, the corresponding reaction proceeded to give thiophthalic anhydride (2u) in a $74 \%$ isolated yield (Scheme 2). 


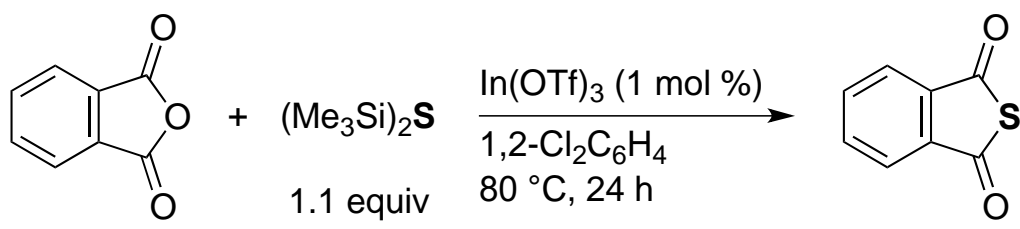

1u
$2 u, 74 \%$

Scheme 2. Reaction of phthalic anhydride.

Although an acyclic ester, the methyl benzoate derivative 1v was not acceptable to the transformation under the optimal conditions; the use of $5 \mathrm{~mol} \%$ of $\operatorname{InI}_{3}$ catalyst at $120{ }^{\circ} \mathrm{C}$ for $20 \mathrm{~h}$ improved the reactivity for the reaction, leading to the expected thioester $2 \mathrm{v}$ in a $38 \%$ isolated yield. Along with the formation of the thioester $2 \mathbf{v}$ in those conditions, the unexpected dithioester $4 \mathbf{v}$ was also isolated in an $18 \%$ yield (Scheme 3 ).

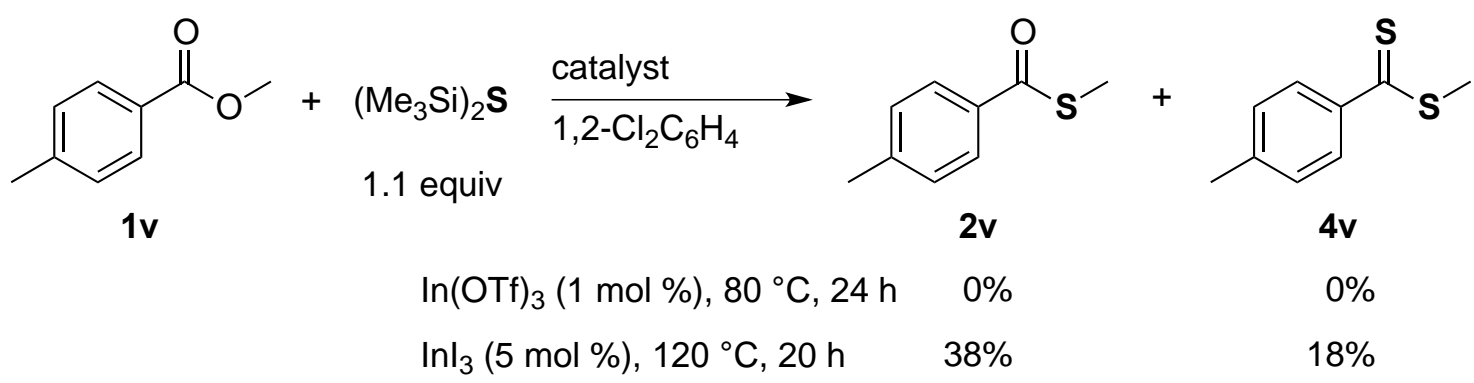

Scheme 3. Reaction of an acyclic ester.

\section{Materials and Methods}

\subsection{General Information}

${ }^{1} \mathrm{H}$ - and ${ }^{13} \mathrm{C}-\mathrm{NMR}$ spectra were recorded on a 300 or $500 \mathrm{MHz}$ spectrometer. Chemical shifts in the ${ }^{1} \mathrm{H}$ - and ${ }^{13} \mathrm{C}$-NMR spectra were reported in ppm relative to the residual solvent peaks, such as those of chloroform $\left(\delta 7.26\right.$ for ${ }^{1} \mathrm{H}$ and $\delta 77.0$ for $\left.{ }^{13} \mathrm{C}\right)$ or of the internal reference tetramethylsilane $(\delta 0.00$ for both ${ }^{1} \mathrm{H}$ and ${ }^{13} \mathrm{C}$ ). High-resolution mass spectra (HRMS) were measured using NBA (3-nitrobenzylalcohol) as a matrix. GC analyses were performed using a DB-5 capillary column $(30 \mathrm{~m} \times 0.25 \mathrm{~mm}$, film thickness $=0.25 \mu \mathrm{m}$ ). Reactions were monitored by TLC analysis of the reaction aliquots. Column chromatography was performed using a silica gel. All indium compounds and hexamethyldisilathiane were commercially available and were used without further purification. 1,2-Dichlorobenzene was

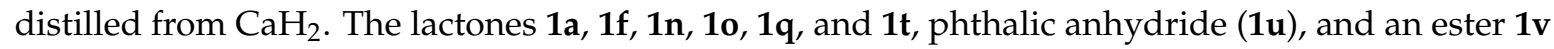
were purchased and used without further purification. The lactones $\mathbf{1 b}$ and $\mathbf{1 l}$ were prepared by the gallium-catalyzed reductive cyclization of keto acids [14]. The lactones $\mathbf{1 c}, \mathbf{1 d}, \mathbf{1 e}, \mathbf{1} \mathbf{g}, \mathbf{1} \mathbf{h}, \mathbf{1} \mathbf{i}, \mathbf{1} \mathbf{j}, \mathbf{1 k}, \mathbf{1 m}$, 1p, 1r, and 1s were prepared via a modified literature method [15].

3.2. General Procedure A for the Indium-Catalyzed Conversion of Lactones or Their Derivatives 1 into Thiolactones $\mathbf{2}$ Using a Disilathiane (In the Case of $\mathbf{1}$ in Solid State at Room Temperature)

To a screw-capped tube, lactone or the derivative $1(0.50 \mathrm{mmol})$ was added. The tube was sealed and moved into a glovebox, then $\operatorname{In}(\mathrm{OTf})_{3}(2.8 \mathrm{mg}, 0.0050 \mathrm{mmol})$ was added. The tube was sealed again and removed from the glovebox. 1,2-Dichlorobenzene $(0.5 \mathrm{~mL})$ and hexamethyldisilathiane $(98.1 \mathrm{mg}, 0.550 \mathrm{mmol})$ were successively added, and after the tube was sealed, the mixture was heated at $80^{\circ} \mathrm{C}$ for $24 \mathrm{~h}$. The resulting mixture was cooled to room temperature and chloroform was added. The mixture was transferred into a round-bottom flask, which was then evaporated under reduced pressure. The crude material was purified by silica gel column chromatography (hexane/EtOAc) followed by gel permeation chromatography (GPC) in some cases. 
3.3. General Procedure B for the Indium-Catalyzed Conversion of Lactones $\mathbf{1}$ into Thiolactones $\mathbf{2}$ Using a Disilathiane (In the Case of $\mathbf{1}$ in Liquid State at Room Temperature)

To a screw-capped tube, $\operatorname{In}(\mathrm{OTf})_{3}(2.8 \mathrm{mg}, 0.0050 \mathrm{mmol})$ was added in a glovebox. The tube was then sealed and removed from the glovebox, and 1,2-dichlorobenzene (0.5 mL), lactone $\mathbf{1}(0.50 \mathrm{mmol})$, and hexamethyldisilathiane $(98.1 \mathrm{mg}, 0.550 \mathrm{mmol})$ were added in this order. After the tube was sealed, the mixture was heated at $80^{\circ} \mathrm{C}$ for $24 \mathrm{~h}$. The resulting mixture was cooled to room temperature and chloroform was added. The mixture was transferred into a round-bottom flask, which was then evaporated under reduced pressure. The crude material was purified by silica gel column chromatography (hexane/EtOAc) followed by gel permeation chromatography (GPC) in some cases.

\subsection{Product Characterization}

Dihydro-5-phenyl-2(3H)-thiophenone (2a) [13]. General procedure A was followed with 5-phenyldihydrofuran-2-one (1a, $80.2 \mathrm{mg})$. Column chromatography (10/1 hexane/EtOAc) afforded 2a as a colorless oil (73.4 mg, 83\%): ${ }^{1} \mathrm{H}-\mathrm{NMR}\left(\mathrm{CDCl}_{3}, 500 \mathrm{MHz}\right) \delta 2.24-2.32\left(\mathrm{~m}, 1 \mathrm{H}, \mathrm{CH}_{2}\right), 2.59-2.82$ $\left(\mathrm{m}, 3 \mathrm{H}, \mathrm{CH}_{2}, \mathrm{CH}_{2}\right), 5.01$ (dd, $\left.J=10.0,5.5 \mathrm{~Hz}, 1 \mathrm{H}, \mathrm{CH}\right), 7.30-7.44(\mathrm{~m}, 5 \mathrm{H}, \mathrm{ArH})$; ${ }^{13} \mathrm{C}-\mathrm{NMR}\left(\mathrm{CDCl}_{3}\right.$, $126 \mathrm{MHz}) \delta 35.0,42.9,54.3,127.4,128.1,128.9,139.4,207.9 ; \mathrm{MS}$ (EI) $m / z(\%) 178$ (M $\left.{ }^{+}, 78\right), 117$ (100).

Dihydro-5-(2-methylphenyl)-2(3H)-thiophenone (2b) [13]. General procedure B was followed with 5-(2-methylphenyl)dihydrofuran-2-one (1b, $89.9 \mathrm{mg})$. Column chromatography (10/1 hexane/EtOAc) afforded $\mathbf{2 b}$ as a colorless oil $(69.7 \mathrm{mg}, 73 \%):{ }^{1} \mathrm{H}-\mathrm{NMR}\left(\mathrm{CDCl}_{3}, 500 \mathrm{MHz}\right) \delta 2.26-2.34\left(\mathrm{~m}, 1 \mathrm{H}, \mathrm{CH}_{2}\right)$, $2.42\left(\mathrm{~s}, 3 \mathrm{H}, \mathrm{CH}_{3}\right), 2.55-2.61\left(\mathrm{~m}, 1 \mathrm{H}, \mathrm{CH}_{2}\right), 2.67-2.74\left(\mathrm{~m}, 1 \mathrm{H}, \mathrm{CH}_{2}\right), 2.78-2.84\left(\mathrm{~m}, 1 \mathrm{H}, \mathrm{CH}_{2}\right), 5.25(\mathrm{dd}$, $J=9.5,5.5 \mathrm{~Hz}, 1 \mathrm{H}, \mathrm{CH}), 7.19-7.27(\mathrm{~m}, 3 \mathrm{H}, \mathrm{ArH}), 7.56(\mathrm{~d}, J=8.0 \mathrm{~Hz}, 1 \mathrm{H}, \mathrm{ArH}) ;{ }^{13} \mathrm{C}-\mathrm{NMR}\left(\mathrm{CDCl}_{3}\right.$, $126 \mathrm{MHz}) \delta 19.5,33.4,42.7,50.3,126.5,126.7,127.8,130.7,135.7,137.2,208.0 ; \mathrm{MS}(\mathrm{EI}) \mathrm{m} / z(\%) 192\left(\mathrm{M}^{+}\right.$, 82), 117 (100).

Dihydro-5-(3-methylphenyl)-2(3H)-thiophenone (2c) [13]. General procedure B was followed with 5-(3-methylphenyl)dihydrofuran-2-one (1c, $92.3 \mathrm{mg})$. Column chromatography (10/1 hexane/EtOAc) afforded $2 \mathrm{c}$ as a dark yellow oil $(81.9 \mathrm{mg}, 82 \%):{ }^{1} \mathrm{H}-\mathrm{NMR}\left(\mathrm{CDCl}_{3}, 500 \mathrm{MHz}\right) \delta 2.23-2.31\left(\mathrm{~m}, 1 \mathrm{H}, \mathrm{CH}_{2}\right)$, $2.37\left(\mathrm{~s}, 3 \mathrm{H}, \mathrm{CH}_{3}\right), 2.57-2.81\left(\mathrm{~m}, 3 \mathrm{H}, \mathrm{CH}_{2}, \mathrm{CH}_{2}\right), 4.97(\mathrm{dd}, J=10.0,5.5 \mathrm{~Hz}, 1 \mathrm{H}, \mathrm{CH}), 7.13(\mathrm{~d}, J=7.5 \mathrm{~Hz}$, $1 \mathrm{H}, \mathrm{ArH}), 7.21-7.28(\mathrm{~m}, 3 \mathrm{H}, \mathrm{ArH}) ;{ }^{13} \mathrm{C}-\mathrm{NMR}\left(\mathrm{CDCl}_{3}, 126 \mathrm{MHz}\right) \delta 21.4,35.0,42.9,54.3,124.4,128.1$, 128.7, 128.9, 138.6, 139.3, 208.0; MS (EI) $m / z(\%) 192\left(\mathrm{M}^{+}, 100\right)$.

Dihydro-5-(4-methylphenyl)-2(3H)-thiophenone (2d) [13]. General procedure A was followed with 5-(4-methylphenyl)dihydrofuran-2-one (1d, $87.5 \mathrm{mg})$. Column chromatography (10/1 hexane/EtOAc) afforded $2 \mathrm{~d}$ as a colorless oil $(61.3 \mathrm{mg}, 64 \%):{ }^{1} \mathrm{H}-\mathrm{NMR}\left(\mathrm{CDCl}_{3}, 500 \mathrm{MHz}\right) \delta 2.21-2.29\left(\mathrm{~m}, 1 \mathrm{H}, \mathrm{CH}_{2}\right)$, $2.35\left(\mathrm{~s}, 3 \mathrm{H}, \mathrm{CH}_{3}\right), 2.56-2.78\left(\mathrm{~m}, 3 \mathrm{H}, \mathrm{CH}_{2}, \mathrm{CH}_{2}\right), 4.96(\mathrm{dd}, J=10.0,5.5 \mathrm{~Hz}, 1 \mathrm{H}, \mathrm{CH}), 7.17(\mathrm{~d}, J=8.0 \mathrm{~Hz}$, $2 \mathrm{H}, \mathrm{ArH}), 7.30(\mathrm{~d}, J=8.0 \mathrm{~Hz}, 2 \mathrm{H}, \mathrm{ArH}) ;{ }^{13} \mathrm{C}-\mathrm{NMR}\left(\mathrm{CDCl}_{3}, 126 \mathrm{MHz}\right) \delta 21.0,35.0,42.9,54.1,127.2$, 129.5, 136.4, 137.9, 208.0; MS (EI) $m / z$ (\%) $192\left(\mathrm{M}^{+}, 82\right), 117$ (100).

Dihydro-5-(2,5-dimethylphenyl)-2(3H)-thiophenone (2e) [13]. General procedure A was followed with 5-(2,5-dimethylphenyl)dihydrofuran-2-one (1e, $94.5 \mathrm{mg})$. Column chromatography (10/1 hexane/EtOAc) afforded $2 \mathrm{e}$ as a yellow oil $(66.6 \mathrm{mg}, 65 \%)$ : ${ }^{1} \mathrm{H}-\mathrm{NMR}\left(\mathrm{CDCl}_{3}, 500 \mathrm{MHz}\right) \delta 2.24-2.31\left(\mathrm{~m}, 1 \mathrm{H}, \mathrm{CH}_{2}\right), 2.33$ $\left(\mathrm{s}, 3 \mathrm{H}, \mathrm{CH}_{3}\right), 2.36\left(\mathrm{~s}, 3 \mathrm{H}, \mathrm{CH}_{3}\right), 2.53-2.58\left(\mathrm{~m}, 1 \mathrm{H}, \mathrm{CH}_{2}\right), 2.65-2.73\left(\mathrm{~m}, 1 \mathrm{H}, \mathrm{CH}_{2}\right), 2.76-2.82(\mathrm{~m}, 1 \mathrm{H}$, $\left.\mathrm{CH}_{2}\right), 5.22(\mathrm{dd}, J=10.0,5.5 \mathrm{~Hz}, 1 \mathrm{H}, \mathrm{CH}), 7.01(\mathrm{~d}, J=7.5 \mathrm{~Hz}, 1 \mathrm{H}, \mathrm{ArH}), 7.07(\mathrm{~d}, J=7.5 \mathrm{~Hz}, 1 \mathrm{H}, \mathrm{ArH})$, 7.36 (s, $1 \mathrm{H}, \mathrm{ArH}) ;{ }^{13} \mathrm{C}-\mathrm{NMR}\left(\mathrm{CDCl}_{3}, 126 \mathrm{MHz}\right) \delta 19.0,21.0,33.4,42.7,50.3,127.1,128.5,130.6,132.5$, 136.2, 136.9, 208.1; MS (EI) $m / z(\%) 206\left(\mathrm{M}^{+}, 85\right), 131$ (100).

Dihydro-5-([1,1'-biphenyl]-4-yl)-2(3H)-thiophenone (2f). General procedure A was followed with 5-[1,1'-Biphenyl]-4-yldihydro-2(3H)-furanone (1f, $96.2 \mathrm{mg})$. Column chromatography (10/1 hexane/EtOAc) and GPC afforded $2 \mathrm{f}$ as a colorless solid $(61.6 \mathrm{mg}, 61 \%)$ : m.p. $120-121{ }^{\circ} \mathrm{C} ;{ }^{1} \mathrm{H}-\mathrm{NMR}$ $\left(\mathrm{CDCl}_{3}, 500 \mathrm{MHz}\right) \delta 2.78-2.36\left(\mathrm{~m}, 1 \mathrm{H}, \mathrm{CH}_{2}\right), 2.63-2.83\left(\mathrm{~m}, 3 \mathrm{H}, \mathrm{CH}_{2}, \mathrm{CH}_{2}\right), 5.05(\mathrm{dd}, J=10.0,5.5 \mathrm{~Hz}, 1 \mathrm{H}$,

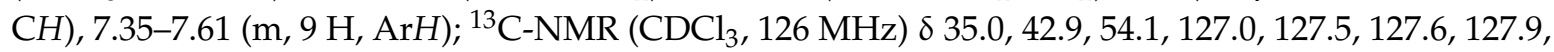


128.8, 138.4, 140.4, 141.1, 207.8; MS (EI) $m / z(\%) 254\left(\mathrm{M}^{+}, 100\right)$; HRMS (EI) calcd for $[\mathrm{M}]^{+}\left(\mathrm{C}_{16} \mathrm{H}_{14} \mathrm{OS}\right)$ $m / z 254.0765$, found 254.0771 .

Dihydro-5-(3-methoxyphenyl)-2(3H)-thiophenone (2g) [13]. General procedure B was followed with 5-(3-methoxyphenyl)dihydrofuran-2-one (1g, $100.9 \mathrm{mg})$. Column chromatography (10/1 hexane/EtOAc) afforded $2 \mathrm{~g}$ as a yellow oil $(86.4 \mathrm{mg}, 79 \%):{ }^{1} \mathrm{H}-\mathrm{NMR}\left(\mathrm{CDCl}_{3}, 500 \mathrm{MHz}\right) \delta 2.21-2.30\left(\mathrm{~m}, 1 \mathrm{H}, \mathrm{CH}_{2}\right)$, 2.59-2.78 (m, $\left.3 \mathrm{H}, \mathrm{CH}_{2}, \mathrm{CH}_{2}\right), 3.81\left(\mathrm{~s}, 3 \mathrm{H}, \mathrm{CH}_{3}\right), 4.96(\mathrm{dd}, J=10.0,5.5 \mathrm{~Hz}, 1 \mathrm{H}, \mathrm{CH}), 6.85(\mathrm{dd}, J=8.0$, $3.0 \mathrm{~Hz}, 1 \mathrm{H}, \mathrm{ArH}), 6.97(\mathrm{~s}, 1 \mathrm{H}, \mathrm{ArH}), 7.00(\mathrm{~d}, J=8.0 \mathrm{~Hz}, 1 \mathrm{H}, \operatorname{ArH}), 7.28(\mathrm{dd}, J=8.0,8.0 \mathrm{~Hz}, 1 \mathrm{H}, \operatorname{Ar} H)$; ${ }^{13} \mathrm{C}-\mathrm{NMR}\left(\mathrm{CDCl}_{3}, 126 \mathrm{MHz}\right) \delta 34.8,42.7,54.2,55.2,113.1,113.3,119.6,129.8,140.9,159.8,207.8$; MS (EI) $m / z(\%) 208\left(\mathrm{M}^{+}, 100\right)$.

Dihydro-5-(4-methoxyphenyl)-2(3H)-thiophenone (2h) [13]. General procedure B was followed with 5-(4-methoxyphenyl)dihydrofuran-2-one (1h, $97.7 \mathrm{mg})$. Column chromatography (10/1 hexane/EtOAc) afforded $2 \mathrm{~h}$ as a colorless solid (23.2 mg, $22 \%)$ : m.p. $74-75{ }^{\circ} \mathrm{C} ;{ }^{1} \mathrm{H}-\mathrm{NMR}\left(\mathrm{CDCl}_{3}, 500 \mathrm{MHz}\right) \delta 2.21-2.29$ (m, $\left.1 \mathrm{H}, \mathrm{CH}_{2}\right), 2.56-2.59\left(\mathrm{~m}, 1 \mathrm{H}, \mathrm{CH}_{2}\right), 2.65-2.80\left(\mathrm{~m}, 2 \mathrm{H}, \mathrm{CH}_{2}, \mathrm{CH}_{2}\right), 3.81\left(\mathrm{~s}, 3 \mathrm{H}, \mathrm{CH}_{3}\right), 4.97(\mathrm{dd}$, $J=10.5,5.5 \mathrm{~Hz}, 1 \mathrm{H}, \mathrm{CH}), 6.90(\mathrm{~d}, J=8.0 \mathrm{~Hz}, 2 \mathrm{H}, \mathrm{ArH}), 7.35(\mathrm{~d}, J=8.0 \mathrm{~Hz}, 2 \mathrm{H}, \mathrm{ArH}) ;{ }^{13} \mathrm{C}-\mathrm{NMR}\left(\mathrm{CDCl}_{3}\right.$,

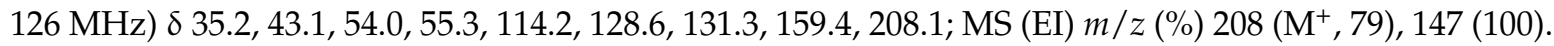

Dihydro-5-(4-fluorophenyl)-2(3H)-thiophenone (2i) [13]. General procedure B was followed with 5-(4-fluorophenyl)dihydrofuran-2-one (1i, $96.3 \mathrm{mg})$. Column chromatography (10/1 hexane/EtOAc) afforded $2 \mathbf{i}$ as a pale green oil $(82.3 \mathrm{mg}, 79 \%):{ }^{1} \mathrm{H}-\mathrm{NMR}\left(\mathrm{CDCl}_{3}, 297 \mathrm{MHz}\right) \delta 2.17-2.29\left(\mathrm{~m}, 1 \mathrm{H}, \mathrm{CH}_{2}\right)$, 2.56-2.82 (m, $\left.3 \mathrm{H}, \mathrm{CH}_{2}, \mathrm{CH}_{2}\right), 4.99(\mathrm{dd}, J=9.8,5.0 \mathrm{~Hz}, 1 \mathrm{H}, \mathrm{CH}), 7.03-7.08(\mathrm{~m}, 2 \mathrm{H}, \mathrm{ArH}), 7.38-7.42(\mathrm{~m}$, $2 \mathrm{H}, \mathrm{ArH}) ;{ }^{13} \mathrm{C}-\mathrm{NMR}\left(\mathrm{CDCl}_{3}, 75 \mathrm{MHz}\right) \delta 35.1,42.8,53.5,115.7\left(\mathrm{~d}, J_{\mathrm{C}-\mathrm{F}}=20.9 \mathrm{~Hz}\right), 129.0\left(\mathrm{~d}, J_{\mathrm{C}-\mathrm{F}}=9.0 \mathrm{~Hz}\right)$, $135.2\left(\mathrm{~d}, J_{\mathrm{C}-\mathrm{F}}=3.7 \mathrm{~Hz}\right), 162.3\left(\mathrm{~d}, J_{\mathrm{C}-\mathrm{F}}=246.8 \mathrm{~Hz}\right), 207.4$; MS (EI) $\mathrm{m} / \mathrm{z}(\%) 196\left(\mathrm{M}^{+}, 72\right), 135(100)$.

Dihydro-5-(4-chlorophenyl)-2(3H)-thiophenone (2j) [13]. General procedure A was followed with 5-(4-chlorophenyl)dihydrofuran-2-one (1j, $98.1 \mathrm{mg})$. Column chromatography (10/1 hexane/EtOAc) afforded $2 \mathbf{j}$ as a colorless solid $(85.5 \mathrm{mg}, 87 \%)$ : m.p. $45-48{ }^{\circ} \mathrm{C} ;{ }^{1} \mathrm{H}-\mathrm{NMR}\left(\mathrm{CDCl}_{3}, 500 \mathrm{MHz}\right) \delta 2.18-2.26$ (m, $\left.1 \mathrm{H}, \mathrm{CH}_{2}\right), 2.59-2.80\left(\mathrm{~m}, 3 \mathrm{H}, \mathrm{CH}_{2}, \mathrm{CH}_{2}\right), 4.97(\mathrm{dd}, J=10.0,5.5 \mathrm{~Hz}, 1 \mathrm{H}, \mathrm{CH}), 7.34-7.35(\mathrm{~m}, 4 \mathrm{H}$, $\mathrm{ArH}) ;{ }^{13} \mathrm{C}-\mathrm{NMR}\left(\mathrm{CDCl}_{3}, 126 \mathrm{MHz}\right) \delta 34.9,42.7,53.5,128.7,129.0,133.8,138.0,207.2 ; \mathrm{MS}$ (EI) $\mathrm{m} / z(\%)$ $214\left(\mathrm{M}^{+}+2,23\right), 212\left(\mathrm{M}^{+}, 63\right), 117(100)$.

Dihydro-5-(4-bromophenyl)-2(3H)-thiophenone (2k) [13]. General procedure A was followed with 5-(4-bromophenyl)dihydrofuran-2-one (1k, $120.1 \mathrm{mg})$. Column chromatography (10/1 hexane/EtOAc) afforded $\mathbf{2 k}$ as a colorless solid $(96.1 \mathrm{mg}, 74 \%)$ : m.p. 58-60 ${ }^{\circ} \mathrm{C} ;{ }^{1} \mathrm{H}-\mathrm{NMR}\left(\mathrm{CDCl}_{3}, 500 \mathrm{MHz}\right) \delta 2.18-2.26$ $\left(\mathrm{m}, 1 \mathrm{H}, \mathrm{CH}_{2}\right), 2.58-2.80\left(\mathrm{~m}, 3 \mathrm{H}, \mathrm{CH}_{2}, \mathrm{CH}_{2}\right), 4.95(\mathrm{dd}, J=10.0,5.5 \mathrm{~Hz}, 1 \mathrm{H}, \mathrm{CH}), 7.30(\mathrm{~d}, J=7.0 \mathrm{~Hz}, 2 \mathrm{H}$, $\mathrm{ArH}), 7.50(\mathrm{~d}, J=7.0 \mathrm{~Hz}, 2 \mathrm{H}, \mathrm{ArH}) ;{ }^{13} \mathrm{C}-\mathrm{NMR}\left(\mathrm{CDCl}_{3}, 126 \mathrm{MHz}\right) \delta 34.9,42.7,53.6,122.0,129.1,132.0$, 138.6, 207.2; MS (EI) $m / z(\%) 258\left(\mathrm{M}^{+}+2,59\right), 256\left(\mathrm{M}^{+}, 58\right), 117(100)$.

Dihydro-5-(5,6,7,8-tetrahydronaphthalen-2-yl)-2(3H)-thiophenone (21) [13]. General procedure B was followed with 5-(5,6,7,8-Tetrahydronaphthalen-2-yl)dihydrofuran-2(3H)-one (11, $96.8 \mathrm{mg})$. Column chromatography (10/1 hexane/EtOAc) and GPC afforded 21 as a colorless oil (37.4 mg, 36\%): ${ }^{1} \mathrm{H}-\mathrm{NMR}$ $\left(\mathrm{CDCl}_{3}, 500 \mathrm{MHz}\right) \delta 1.78-1.81\left(\mathrm{~m}, 4 \mathrm{H}, \mathrm{CH}_{2}\right), 2.23-2.31\left(\mathrm{~m}, 1 \mathrm{H}, \mathrm{CH}_{2}\right), 2.55-2.80\left(\mathrm{~m}, 7 \mathrm{H}, \mathrm{CH}_{2}, \mathrm{CH}_{2}\right)$, $4.94(\mathrm{dd}, J=10.5,5.5 \mathrm{~Hz}, 1 \mathrm{H}, \mathrm{CH}), 7.07(\mathrm{~d}, J=8.0 \mathrm{~Hz} 1 \mathrm{H}, \mathrm{ArH}) 7.12-7.15(\mathrm{~m}, 2 \mathrm{H}, \mathrm{ArH}) ;{ }^{13} \mathrm{C}-\mathrm{NMR}$ $\left(\mathrm{CDCl}_{3}, 126 \mathrm{MHz}\right)$ 8 23.0, 23.1, 29.1, 29.4, 35.0, 43.0, 54.3, 124.4, 128.1, 129.6, 136.4, 137.3, 137.7, 208.3; MS (EI) $m / z(\%) 232\left(\mathbf{M}^{+}, 100\right)$.

Dihydro-5-(thiophen-2-yl)-2(3H)-thiophenone (2m). General procedure B was followed with dihydro-5-(3-thienyl)-2(3H)-furanone $(\mathbf{1 m}, 89.0 \mathrm{mg})$. Column chromatography (10/1 hexane/EtOAc) afforded $2 \mathrm{~m}$ as a light green oil $(22.3 \mathrm{mg}, 23 \%):{ }^{1} \mathrm{H}-\mathrm{NMR}\left(\mathrm{CDCl}_{3}, 500 \mathrm{MHz}\right) \delta 2.34-2.42\left(\mathrm{~m}, 1 \mathrm{H}, \mathrm{CH}_{2}\right)$, 2.65-2.85 (m, $\left.3 \mathrm{H}, \mathrm{CH}_{2}, \mathrm{CH}_{2}\right), 5.29(\mathrm{dd}, J=7.5,5.5 \mathrm{~Hz}, 1 \mathrm{H}, \mathrm{CH}), 6.97(\mathrm{dd}, J=5.0,3.5 \mathrm{~Hz}, 1 \mathrm{H}, \mathrm{ArH})$, $7.07(\mathrm{~d}, J=3.5 \mathrm{~Hz}, 1 \mathrm{H}, \mathrm{ArH}), 7.27(\mathrm{dd}, J=5.0,3.5 \mathrm{~Hz}, 1 \mathrm{H}, \mathrm{ArH}) ;{ }^{13} \mathrm{C}-\mathrm{NMR}\left(\mathrm{CDCl}_{3}, 126 \mathrm{MHz}\right) \delta 35.6$, 42.3, 49.1, 125.3, 125.6, 126.9, 143.7, 207.0; MS (EI) $m / z(\%) 184\left(\mathrm{M}^{+}, 64\right), 123$ (100); HRMS (EI) calcd for $[\mathrm{M}]^{+}\left(\mathrm{C}_{8} \mathrm{H}_{8} \mathrm{OS}_{2}\right) m / z$ 184.0017, found 184.0011. 
Thiophthalide (2o) [16]. General procedure A was followed with phthalide (10, $67.6 \mathrm{mg})$. Column chromatography (10/1 hexane/EtOAc) afforded 20 as a colorless solid $(26.8 \mathrm{mg}, 18 \%)$ : m.p. $68-70{ }^{\circ} \mathrm{C}$; ${ }^{1} \mathrm{H}-\mathrm{NMR}\left(\mathrm{CDCl}_{3}, 500 \mathrm{MHz}\right) \delta 4.48\left(\mathrm{~s}, 2 \mathrm{H}, \mathrm{CH}_{2}\right), 7.48(\mathrm{dd}, J=7.5,7.5 \mathrm{~Hz}, 1 \mathrm{H}, \mathrm{ArH}), 7.55(\mathrm{~d}, J=7.0 \mathrm{~Hz}$, $1 \mathrm{H}, \mathrm{ArH}), 7.63(\mathrm{dd}, J=7.5,7.5 \mathrm{~Hz}, 1 \mathrm{H}, \mathrm{ArH}), 7.85(\mathrm{~d}, J=7.5 \mathrm{~Hz}, 1 \mathrm{H}, \mathrm{ArH}) ;{ }^{13} \mathrm{C}-\mathrm{NMR}\left(\mathrm{CDCl}_{3}, 126 \mathrm{MHz}\right)$

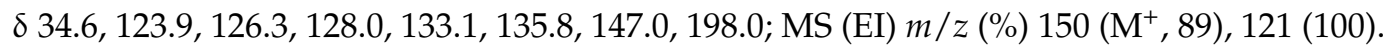

3-Phenyl-benzo[c]thiophen-1(3H)-one (2p) [13]. General procedure A was followed with 3-phenylisobenzofuran-1-one (1p, $105.9 \mathrm{mg})$. Column chromatography (10/1 hexane/EtOAc) afforded $2 \mathrm{p}$ as a pale yellow solid $(7.5 \mathrm{mg}, 85 \%)$ : m.p. $87-88^{\circ} \mathrm{C} ;{ }^{1} \mathrm{H}-\mathrm{NMR}\left(\mathrm{CDCl}_{3}, 500 \mathrm{MHz}\right) \delta 5.91(\mathrm{~s}, 1 \mathrm{H}, \mathrm{CH})$, 7.25-7.36 (m, $6 \mathrm{H}, \mathrm{ArH}), 7.48(\mathrm{dd}, J=7.5,7.5 \mathrm{~Hz}, 1 \mathrm{H}, \mathrm{ArH}), 7.56(\mathrm{dd}, J=7.5,7.5 \mathrm{~Hz}, 1 \mathrm{H}, \mathrm{ArH}), 7.86$ $(\mathrm{d}, J=7.5 \mathrm{~Hz}, 1 \mathrm{H}, \mathrm{ArH}) ;{ }^{13} \mathrm{C}-\mathrm{NMR}\left(\mathrm{CDCl}_{3}, 126 \mathrm{MHz}\right) \delta 54.6,123.6,126.6,128.29,128.33,128.4,129.1$, 133.6, 135.7, 138.8, 151.2, 197.2; MS (EI) $m / z(\%) 226\left(\mathbf{M}^{+}, 100\right)$.

Tetrahydro-6-phenyl-2H-benzothiopyran-2-one (2q) [13]. General procedure A was followed with tetrahydro-6-phenyl-2H-pyran-2-one (1q, $89.3 \mathbf{m g})$. Colum chromatography (10/1 hexane/EtOAc) afforded $2 q$ as an orange oil $(9.7 \mathrm{mg}, 10 \%)$ : ${ }^{1} \mathrm{H}-\mathrm{NMR}\left(\mathrm{CDCl}_{3}, 500 \mathrm{MHz}\right) \delta 1.94-2.10\left(\mathrm{~m}, 2 \mathrm{H}, \mathrm{CH}_{2}\right)$, 2.14-2.19 (m, $\left.1 \mathrm{H}, \mathrm{CH}_{2}\right), 2.37-2.41\left(\mathrm{~m}, 1 \mathrm{H}, \mathrm{CH}_{2}\right), 2.57-2.43\left(\mathrm{~m}, 1 \mathrm{H}, \mathrm{CH}_{2}\right), 2.59-2.78\left(\mathrm{~m}, 1 \mathrm{H}, \mathrm{CH}_{2}\right), 4.65$ $(\mathrm{dd}, J=11.0,4.0 \mathrm{~Hz}, 1 \mathrm{H}, \mathrm{CH}), 7.29-7.32(\mathrm{~m}, 1 \mathrm{H}, \mathrm{ArH}), 7.35-7.39(\mathrm{~m}, 4 \mathrm{H}, \mathrm{ArH}) ;{ }^{13} \mathrm{C}-\mathrm{NMR}\left(\mathrm{CDCl}_{3}\right.$, $126 \mathrm{MHz}) \delta 22.7,32.4,40.5,50.5,127.7,128.1,128.9,140.4,201.3 ; \mathrm{MS}$ (EI) $m / z(\%) 192\left(\mathrm{M}^{+}, 59\right), 104$ (100).

Tetrahydro-6-(4-methylphenyl)-2H-thiopyran-2-one (2r). General procedure A was followed with tetrahydro-6-(4-methylphenyl)-2H-Pyran-2-one (1r, $92.3 \mathrm{mg})$. Column chromatography (10/1 hexane/EtOAc) and GPC afforded $2 \mathrm{r}$ as a colorless oil $(9.5 \mathrm{mg}, 9 \%):{ }^{1} \mathrm{H}-\mathrm{NMR}\left(\mathrm{CDCl}_{3}, 500 \mathrm{MHz}\right)$ 反 1.93-2.07 (m, $\left.2 \mathrm{H}, \mathrm{CH}_{2}\right), 2.15-2.17\left(\mathrm{~m}, 1 \mathrm{H}, \mathrm{CH}_{2}\right), 2.35\left(\mathrm{~s}, 3 \mathrm{H}, \mathrm{CH}_{3}\right), 2.37-2.44\left(\mathrm{~m}, 1 \mathrm{H}, \mathrm{CH}_{2}\right), 2.72-2.76$ $\left(\mathrm{m}, 1 \mathrm{H}, \mathrm{CH}_{2}\right), 4.61(\mathrm{dd}, J=11.0,3.5 \mathrm{~Hz}, 1 \mathrm{H}, \mathrm{CH}), 7.17(\mathrm{~d}, J=7.5 \mathrm{~Hz}, 2 \mathrm{H}, \operatorname{ArH}), 7.26(\mathrm{~d}, J=7.5 \mathrm{~Hz}, 2 \mathrm{H}$, $\mathrm{ArH}) ;{ }^{13} \mathrm{C}-\mathrm{NMR}\left(\mathrm{CDCl}_{3}, 126 \mathrm{MHz}\right) \delta 21.1,22.7,32.4,40.5,50.2,127.5,129.5,137.4,137.9,201.6$; MS (EI) $m / z(\%) 206\left(\mathrm{M}^{+}, 62\right), 118$ (100); HRMS (EI) calcd for [M] ${ }^{+}\left(\mathrm{C}_{12} \mathrm{H}_{14} \mathrm{OS}\right) \mathrm{m} / z$ 206.0765, found 206.0766.

6-(4-Chlorophenyl)tetrahydro-2H-thiopyran-2-one (2s). General procedure A was followed with 6-(4-chlorophenyl)tetrahydro-2H-pyran-2-one (1s, $107.5 \mathrm{mg})$. Column chromatography (10/1 hexane/EtOAc) and GPC afforded $2 \mathrm{~s}$ as a colorless oil $(4.8 \mathrm{mg}, 4 \%):{ }^{1} \mathrm{H}-\mathrm{NMR}\left(\mathrm{CDCl}_{3}, 500 \mathrm{MHz}\right) \delta$ 1.93-2.02 (m, $\left.2 \mathrm{H}, \mathrm{CH}_{2}\right), 2.04-2.19\left(\mathrm{~m}, 1 \mathrm{H}, \mathrm{CH}_{2}\right), 2.34-2.39\left(\mathrm{~m}, 1 \mathrm{H}, \mathrm{CH}_{2}\right), 2.56-2.63\left(\mathrm{~m}, 1 \mathrm{H}, \mathrm{CH}_{2}\right)$, 2.73-2.78 (m, $\left.1 \mathrm{H}, \mathrm{CH}_{2}\right), 4.62(\mathrm{dd}, J=11.0,4.5 \mathrm{~Hz}, 1 \mathrm{H}, \mathrm{CH}), 7.30-7.35(\mathrm{~m}, 4 \mathrm{H}, \mathrm{ArH}) ;{ }^{13} \mathrm{C}-\mathrm{NMR}\left(\mathrm{CDCl}_{3}\right.$, $126 \mathrm{MHz}) \delta 22.6,32.4,40.5,49.7,129.0,129.1,133.9,139.0,200.8$; MS (EI) $m / z$ (\%) $226\left(\mathrm{M}^{+}+2,20\right), 210$ $\left(\mathrm{M}^{+}, 23\right), 138$ (100); HRMS (EI) calcd for $[\mathrm{M}]^{+}\left(\mathrm{C}_{11} \mathrm{H}_{11} \mathrm{OSCl}\right) \mathrm{m} / z$ 226.0219, found 226.0241.

1,4-Dihydro-3H-2-benzothiopyran-3-one (2t) [13]. General procedure A was followed with 1,4-dihydro-3H-2-benzopyran-3-one (1t, $74.3 \mathrm{mg})$. Column chromatography (10/1 hexane/EtOAc) afforded $2 \mathbf{t}$ as a pale yellow solid $(40.9 \mathrm{mg}, 50 \%)$ : m.p. $90-93{ }^{\circ} \mathrm{C} ;{ }^{1} \mathrm{H}-\mathrm{NMR}\left(\mathrm{CDCl}_{3}, 500 \mathrm{MHz}\right) \delta$ $3.79\left(\mathrm{~s}, 2 \mathrm{H}, \mathrm{CH}_{2}\right), 4.22\left(\mathrm{~s}, 2 \mathrm{H}, \mathrm{CH}_{2}\right), 7.21-7.32(\mathrm{~m}, 4 \mathrm{H}, \mathrm{ArH}) ;{ }^{13} \mathrm{C}-\mathrm{NMR}\left(\mathrm{CDCl}_{3}, 126 \mathrm{MHz}\right) \delta 34.2,49.2$, 126.6, 127.4, 128.0, 128.7, 133.7, 134.2, 202.9; MS (EI) $m / z$ (\%) $164\left(\mathrm{M}^{+}, 14\right), 104$ (100).

Phthalic thioanhydride (2u) [17]. General procedure A was followed with phthalic anhydride (1u, $77.3 \mathbf{m g})$. Column chromatography (10/1 hexane/EtOAc) afforded $\mathbf{2} \mathbf{u}$ as a yellow solid (56.1 $\mathrm{mg}, 74 \%)$ : m.p. 68-70 ${ }^{\circ} \mathrm{C} ;{ }^{1} \mathrm{H}-\mathrm{NMR}\left(\mathrm{CDCl}_{3}, 500 \mathrm{MHz}\right) \delta 7.81-7.83(\mathrm{~m}, 2 \mathrm{H}, \mathrm{ArH}), 7.97-7.99(\mathrm{~m}, 2 \mathrm{H}, \mathrm{ArH}) ;{ }^{13} \mathrm{C}-\mathrm{NMR}$ $\left(\mathrm{CDCl}_{3}, 126 \mathrm{MHz}\right) \delta 123.8,135.0,138.7,189.8$; MS (EI) $\mathrm{m} / z(\%) 164\left(\mathrm{M}^{+}, 100\right)$.

S-Methyl 4-methylbenzothioate (2v). General procedure A was followed with methyl 4-methylbenzoate (1v, $75.0 \mathrm{mg})$. Column chromatography (100/1 hexane/EtOAc) afforded $2 \mathbf{v}$ as a red oil $(31.8 \mathrm{mg}, 38 \%)$ : ${ }^{1} \mathrm{H}-\mathrm{NMR}\left(\mathrm{CDCl}_{3}, 500 \mathrm{MHz}\right) \delta 2.40\left(\mathrm{~s}, 3 \mathrm{H}, \mathrm{CH}_{3}\right), 2.46\left(\mathrm{~s}, 3 \mathrm{H}, \mathrm{CH}_{3}\right), 7.24(\mathrm{~d}, J=14.5 \mathrm{~Hz}, 2 \mathrm{H}, \mathrm{ArH}), 7.87$ $(\mathrm{d}, J=14.5 \mathrm{~Hz}, 2 \mathrm{H}, \mathrm{ArH}) ;{ }^{13} \mathrm{C}-\mathrm{NMR}\left(\mathrm{CDCl}_{3}, 126 \mathrm{MHz}\right) \delta 11.6,21.7,127.2,129.2,134.5,144.1,192.1$; MS (EI) $m / z(\%) 166\left(\mathbf{M}^{+}, 5\right), 119(100)$.

Methyl 4-methylbenzodithioate (4v) [18]. General procedure A was followed with methyl 4-methylbenzoate $(\mathbf{1 v}, 75.0 \mathrm{mg})$. Column chromatography (100/1 hexane/EtOAc) afforded $4 \mathbf{v}$ as an orange oil $(15.9 \mathrm{mg}$, 
18\%): ${ }^{1} \mathrm{H}-\mathrm{NMR}\left(\mathrm{CDCl}_{3}, 500 \mathrm{MHz}\right) \delta 2.38\left(\mathrm{~s}, 3 \mathrm{H}, \mathrm{CH}_{3}\right), 2.77\left(\mathrm{~s}, 3 \mathrm{H}, \mathrm{CH}_{3}\right), 7.18(\mathrm{~d}, J=13.5 \mathrm{~Hz}, 2 \mathrm{H}$, $\mathrm{ArH}), 7.94(\mathrm{~d}, J=13.5 \mathrm{~Hz}, 2 \mathrm{H}, \mathrm{ArH}) ;{ }^{13} \mathrm{C}-\mathrm{NMR}\left(\mathrm{CDCl}_{3}, 126 \mathrm{MHz}\right) \delta 20.5,21.5,126.8,129.0,142.6,143.2$, 228.8; MS (EI) $m / z(\%) 182\left(\mathrm{M}^{+}, 22\right), 135(100)$.

5-[1,1'-Biphenyl]-4-yldihydro-2(3H)-furanone (1f). A colorless solid: m.p. $100-102{ }^{\circ} \mathrm{C} ;{ }^{1} \mathrm{H}-\mathrm{NMR}\left(\mathrm{CDCl}_{3}\right.$, $500 \mathrm{MHz}) \delta 2.19-2.29\left(\mathrm{~m}, 1 \mathrm{H}, \mathrm{CH}_{2}\right), 2.66-2.72\left(\mathrm{~m}, 3 \mathrm{H}, \mathrm{CH}_{2}\right), 5.54-5.57(\mathrm{~m}, 1 \mathrm{H}, \mathrm{CH}), 7.35-7.46(\mathrm{~m}, 5 \mathrm{H}$, $\mathrm{ArH}), 7.58-7.62(\mathrm{~m}, 4 \mathrm{H}, \mathrm{ArH}) ;{ }^{13} \mathrm{C}-\mathrm{NMR}\left(\mathrm{CDCl}_{3}, 126 \mathrm{MHz}\right) \delta 29.0,30.9,81.0,125.8,127.1,127.45,127.53$, 128.8, 138.3, 140.4, 141.4, 176.9; MS (EI) $m / z(\%) 238\left(\mathrm{M}^{+}, 100\right)$; HRMS (EI) calcd for $[\mathrm{M}]^{+}\left(\mathrm{C}_{16} \mathrm{H}_{14} \mathrm{O}_{2}\right)$ $\mathrm{m} / \mathrm{z} 238.0994$, found 238.1002 .

Tetrahydro-6-(4-methylphenyl)-2H-pyran-2-one (1r). A colorless solid: m.p. $81-83{ }^{\circ} \mathrm{C} ;{ }^{1} \mathrm{H}-\mathrm{NMR}\left(\mathrm{CDCl}_{3}\right.$, $500 \mathrm{MHz}) \delta 1.80-1.88\left(\mathrm{~m}, 1 \mathrm{H}, \mathrm{CH}_{2}\right), 1.92-1.98\left(\mathrm{~m}, 2 \mathrm{H}, \mathrm{CH}_{2}\right), 2.09-2.14\left(\mathrm{~m}, 1 \mathrm{H}, \mathrm{CH}_{2}\right), 2.34(\mathrm{~s}, 3 \mathrm{H}$, $\left.\mathrm{CH}_{3}\right), 2.51-2.56\left(\mathrm{~m}, 1 \mathrm{H}, \mathrm{CH}_{2}\right), 2.57-2.71\left(\mathrm{~m}, 1 \mathrm{H}, \mathrm{CH}_{2}\right), 5.30(\mathrm{dd}, J=10.5,3.5 \mathrm{~Hz}, 1 \mathrm{H}, \mathrm{CH}), 7.17(\mathrm{~d}$, $J=7.5 \mathrm{~Hz}, 2 \mathrm{H}, \mathrm{ArH}), 7.22(\mathrm{~d}, J=7.5 \mathrm{~Hz}, 2 \mathrm{H}, \mathrm{ArH}) ;{ }^{13} \mathrm{C}-\mathrm{NMR}\left(\mathrm{CDCl}_{3}, 126 \mathrm{MHz}\right) \delta 18.4,21.0,29.3,30.3$, $81.5,125.5,129.1,136.6,137.8,171.4 ; \mathrm{MS}(\mathrm{EI}) \mathrm{m} / z(\%) 190\left(\mathrm{M}^{+}, 42\right), 118(100)$; HRMS (EI) calcd for [M] ${ }^{+}$ $\left(\mathrm{C}_{12} \mathrm{H}_{14} \mathrm{O}_{2}\right) \mathrm{m} / z$ 190.0994, found 190.0995 .

Tetrahydro-6-(4-chlorophenyl)-2H-pyran-2-one (1s). An orange solid: m.p. 91-98 ${ }^{\circ} \mathrm{C} ;{ }^{1} \mathrm{H}-\mathrm{NMR}\left(\mathrm{CDCl}_{3}\right.$, $500 \mathrm{MHz}) \delta 1.77-1.85\left(\mathrm{~m}, 1 \mathrm{H}, \mathrm{CH}_{2}\right), 1.96-2.01\left(\mathrm{~m}, 2 \mathrm{H}, \mathrm{CH}_{2}\right), 2.12-2.16\left(\mathrm{~m}, 1 \mathrm{H}, \mathrm{CH}_{2}\right), 2.53-2.60(\mathrm{~m}, 1 \mathrm{H}$, $\left.\mathrm{CH}_{2}\right), 2.67-2.73\left(\mathrm{~m}, 1 \mathrm{H}, \mathrm{CH}_{2}\right), 5.32(\mathrm{dd}, \mathrm{J}=10.5,3.0 \mathrm{~Hz}, 1 \mathrm{H}, \mathrm{CH}), 7.28(\mathrm{~d}, J=8.5 \mathrm{~Hz}, 2 \mathrm{H}, \mathrm{ArH}), 7.34$ $(\mathrm{d}, J=8.5 \mathrm{~Hz}, 2 \mathrm{H}, \mathrm{ArH}) ;{ }^{13} \mathrm{C}-\mathrm{NMR}\left(\mathrm{CDCl}_{3}, 126 \mathrm{MHz}\right) \delta 18.4,29.3,30.4,80.8,127.0,128.6,133.9,138.2$, 171.0; MS (EI) m/z (\%) $212\left(\mathrm{M}^{+}+2,7\right), 210\left(\mathrm{M}^{+}, 23\right), 70(100)$; HRMS (EI) calcd for $[\mathrm{M}]^{+}\left(\mathrm{C}_{11} \mathrm{H}_{11} \mathrm{O}_{2} \mathrm{Cl}_{)}\right.$ $m / z 210.0448$, found 210.0449 .

\section{Conclusions}

An indium-catalyzed formation of thiolactones from lactones and a disilathiane was developed. A disilathiane was found to be a novel and an effective sulfur source for this type of conversion, and a wide range of lactone derivatives were successfully converted into the corresponding thiolactones.

Supplementary Materials: The following are available online: ${ }^{1} \mathrm{H}$ and ${ }^{13} \mathrm{C}-\mathrm{NMR}$ spectra of $\mathbf{2 a}-\mathbf{2 m} ; \mathbf{2 o - 2 v} ; \mathbf{4 v} ; \mathbf{1 f}$; 1r and 1s.

Author Contributions: N.S. conceived and designed the experiments; K.T. and S.H. performed the experiments; Y.O., K.T. and S.H. analyzed the data; Y.O. and N.S. wrote the paper.

Acknowledgments: The authors deeply thank Shin-Etsu Chemical Co., Ltd., for the gift of hydrosilanes and chlorosilanes.

Conflicts of Interest: The authors declare no conflict of interest.

\section{References}

1. Jones, B.A.; Bradshaw, J.S. Synthesis and reduction of thiocarboxylic O-esters. Chem. Rev. 1984, 84, 17-30. [CrossRef]

2. Beletskaya, I.P.; Ananikov, V.P. Transition-metal-catalyzed C-S, C-Se, and C-Te bond formation via CROSS-coupling and atom-economic addition reactions. Chem. Rev. 2011, 111, 1596-1636. [CrossRef] [PubMed]

3. Liu, H.; Jiang, X. Transfer of sulfur: from simple to diverse. Chem. Asian J. 2013, 8, 2546-2563. [CrossRef] [PubMed]

4. Shen, C.; Zhang, P.; Sun, Q.; Bai, S.; Hor, T.S.A.; Liu, X. Recent advances in C-S bond formation via C-H bond functionalization and decarboxylation. Chem. Soc. Rev. 2015, 44, 291-314. [CrossRef] [PubMed]

5. Ghaderi, A. Advances in transition-metal catalyzed thioetherification reactions of aromatic compounds. Tetrahedron 2016, 72, 4758-4782. [CrossRef]

6. Nguyen, T.B. Recent advances in organic reactions involving elemental sulfur. Adv. Synth. Catal. 2017, 359, 1066-1130. [CrossRef] 
7. Ogiwara, Y.; Maeda, H.; Sakai, N. Copper-catalyzed production of diaryl sulfides using aryl Iodides and a disilathiane. Synlett. 2018, 29, 655-657. [CrossRef]

8. Capozzi, F.; Capozzi, G.; Menichetti, S. Thiiranes: one-pot synthesis from alkenes, and catalytic desulphurization. Tetrahedron Lett. 1988, 29, 4177-4180. [CrossRef]

9. Lin, P.-Y.; Ku, W.-S.; Shiao, M.-J. A new method for converting nitriles into primary thioamides by sodium trimethylsilanethiolate. Synthesis 1992, 1219-1220. [CrossRef]

10. Tsay, S.-C.; Yep, G.L.; Chen, B.-L.; Lin, L.C.; Hwu, J.R. Direct synthesis of diallyl sulfides from allyl alcohols and hexamethyldisilathiane. Tetrahedron 1993, 49, 8969-8976. [CrossRef]

11. Degl'Innocenti, A.; Capperucci, A. Organosilane-induced synthesis and functionalization of sulfur-containing compounds. Eur. J. Org. Chem. 2000, 2171-2186. [CrossRef]

12. Dávila, M.F.C.; Schneider, J.P.; Godard, A.; Hazelard, D.; Compain, P. One-pot, highly stereoselective synthesis of dithioacetal- $\alpha, \alpha$-diglycosides. Molecules 2018, 23, 914. [CrossRef] [PubMed]

13. Sakai, N.; Horikawa, S.; Ogiwara, Y. Indium-catalyzed direct conversion of lactones into thiolactones and selenolactones in the presence of elemental sulfur and selenium. Synthesis 2018, 50, 565-574. [CrossRef]

14. Sakai, N.; Horikawa, S.; Ogiwara, Y. Gallium-catalyzed reductive lactonization of $\gamma$-keto acids with a hydrosilane. RSC Adv. 2016, 6, 81763-81766. [CrossRef]

15. Grover, H.K.; Emmett, M.R.; Kerr, M.A. $\gamma$-Substituted butanolides from cyclopropane hemimalonates: An expedient synthesis of natural (R)-dodecan-4-olide. Org. Lett. 2013, 15, 4838-4841. [CrossRef] [PubMed]

16. Dowd, P.; Weber, W. 3-Thiolen-2-one. A useful dienophile. J. Org. Chem. 1982, 47, 4777-4779. [CrossRef]

17. Murai, T.; Asai, F. Three-component coupling reactions of thioformamides with organolithium and grignard reagents leading to formation of tertiary amines and a thiolating agent. J. Am. Chem. Soc. 2007, 129, 780-781. [CrossRef] [PubMed]

18. Kato, S.; Hori, A.; Mizuta, M.; Katada, T.; Ishihara, H.; Fujieda, K.; Ikebe, Y. On carbon-13 spectra of thioand dithiocarboxylic acids triorgano group 14 metal esters. J. Organomet. Chem. 1991, 420, 13-22. [CrossRef]

Sample Availability: Samples of the all compounds are available from the authors.

(C) 2018 by the authors. Licensee MDPI, Basel, Switzerland. This article is an open access article distributed under the terms and conditions of the Creative Commons Attribution (CC BY) license (http://creativecommons.org/licenses/by/4.0/). 